\title{
Bone Marrow-Derived Progenitor Cells Are Functionally Impaired in Ischemic Heart Disease
}

\author{
Evelien Nollet ${ }^{1,2}$ • Vicky Y. Hoymans ${ }^{1,2}$ - Inez R. Rodrigus ${ }^{3}$ - Dina De Bock ${ }^{3}$ • \\ Marc Dom $^{4}$ • Bruno Vanassche ${ }^{5}$ Viviane O. M. Van Hoof ${ }^{6,7} \cdot$ Nathalie Cools $^{8}$. \\ Katrijn Van Ackeren ${ }^{1,2}$. Kristien Wouters ${ }^{9}$ - Katrien Vermeulen ${ }^{10}$. \\ Christiaan J. Vrints ${ }^{1,2,11}$ • Emeline M. Van Craenenbroeck ${ }^{1,2,11}$
}

Received: 12 April 2016/Accepted: 7 July 2016/Published online: 25 July 2016

(C) Springer Science+Business Media New York 2016

\begin{abstract}
To determine whether the presence of ischemic heart disease (IHD) per se, or rather the co-presence of heart failure $(\mathrm{HF})$, is the primum movens for less effective stem cell products in autologous stem cell therapy, we assessed numbers and function of bone marrow (BM)-derived progenitor cells in patients with coronary artery disease $(n=17)$, HF due to ischemic cardiomyopathy $(n=8)$, non-ischemic HF $(n=7)$, and control subjects $(n=11)$. Myeloid and erythroid differentiation capacity of BM-derived mononuclear cells was impaired in patients with underlying IHD but not with non-ischemic HF. Migration capacity decreased with increasing IHD severity. Hence, IHD, with or without associated cardiomyopathy, is an important determinant of progenitor cell function. No depletion of hematopoietic and endothelial progenitor cells (EPC) within the BM was observed, while circulating EPC numbers were increased in the presence of IHD, suggesting active recruitment. The observed
\end{abstract}

Associate Editor Lorrie Kirshenbaum oversaw the review of this article

Electronic supplementary material The online version of this article (doi:10.1007/s12265-016-9707-z) contains supplementary material, which is available to authorized users.

Evelien Nollet

Evelien.Nollet@uantwerpen.be

1 Laboratory of Cellular and Molecular Cardiology, Department of Cardiology, Antwerp University Hospital, Antwerp, Belgium

2 Cardiovascular Diseases, Department of Translational Pathophysiological Research, University of Antwerp, Antwerp, Belgium

3 Department of Cardiac Surgery, Antwerp University Hospital, Antwerp, Belgium

4 Department of Oral and Maxillofacial Surgery, General Hospital Sint-Maarten, Duffel, Belgium

5 Department of Oral and Maxillofacial Surgery, General Hospital Monica, Antwerp, Belgium myelosuppression was not driven by inflammation and thus other mechanisms are at play.

Keywords Bone marrow · Progenitor cells · Heart failure · Inflammation · Ischemic heart disease

$\begin{array}{ll}\text { Abbreviations } \\ \text { BFU-E } & \text { Erythroid burst-forming units } \\ \text { BM } & \text { Bone marrow } \\ \text { CAD } & \text { Coronary artery disease } \\ \text { EPC } & \text { Endothelial progenitor cell } \\ \text { GM-CFU } & \text { Granulocyte-macrophage colony-forming units } \\ \text { HF } & \text { Heart failure } \\ \text { HPC } & \text { Hematopoietic progenitor cell } \\ \text { ICM } & \text { Ischemic cardiomyopathy }\end{array}$

\section{Abbreviations}

$\mathrm{BFU}$

Bone marrow

Coronary artery disease

Cor

位

Hematopoietic progenitor cell
6 Department of Clinical Chemistry, Antwerp University Hospital, Antwerp, Belgium

7 Biochemistry, Department of Translational Pathophysiological Research, University of Antwerp, Antwerp, Belgium

8 Laboratory of Experimental Hematology, Vaccine and Infectious Disease Institute, University of Antwerp, Antwerp, Belgium

9 Department of Scientific Coordination and Biostatistics, Antwerp University Hospital, Antwerp, Belgium

10 Laboratory of Hematology, Antwerp University Hospital, Antwerp, Belgium

11 Department of Cardiology, Antwerp University Hospital, Antwerp, Belgium 


$\begin{array}{ll}\text { IHD } & \text { Ischemic heart disease } \\ \text { MNC } & \text { Mononuclear cells } \\ \text { LVEF } & \text { Left ventricular ejection fraction } \\ \text { PB } & \text { Peripheral blood }\end{array}$

\section{Introduction}

More than a decade ago, the use of autologous bone marrow (BM)-derived stem cell transplantation was introduced as promising therapy to regenerate infarcted myocardium [1]. Convincing animal data set the stage for thoughtful clinical trials, testing this therapy as a "proof-of-concept." Particularly, the total non-selected BM-derived mononuclear cell (MNC) fractions, but also mesenchymal stem cells, hematopoietic, and endothelial progenitor cells, were studied. Despite the heterogeneity in terms of stem/progenitor cell type, as well as dosing and route of administration, several meta-analyses in the field confirmed its safety and beneficial impact on left ventricular ejection fraction (LVEF), infarct size, and myocardial perfusion $[2,3]$. These benefits were most likely mediated by paracrine actions of injected cells in ischemic tissue, i.e., stimulation of angiogenesis, inhibition of apoptosis of endothelial cells and cardiomyocytes, activation of cardiac stem cells, and recruitment of additional progenitor cells [4].

Despite the initial success of autologous stem cell therapy in the setting of acute myocardial infarction, the outcomes in patients with chronic ischemic heart disease (IHD) and ischemic cardiomyopathy were rather disappointing [5, 6]. Clinical trials reported a mild cardiac improvement in LVEF of $\pm 3 \%$ in patients with IHD, irrespective of reduced LVEF at baseline, compared to the placebo group 3-6 months after intracoronary BM-MNC administration [7, 8].

For patients with dilated cardiomyopathy, results were conflicting: the INTRACELL trial did not find any significant increase in LVEF 3 months after intramyocardial injection of BM-MNC compared to the control group [9]. In contrast, the REGENERATE-DCM trial reported a $5 \%$ increase in LVEF 3 months after intracoronary administration of BM cells in patients with non-ischemic heart failure (HF) compared to baseline [10]. Hence, researchers were drawn back to the bench to investigate why results have proved inferior to what was anticipated from clinical studies.

At present, there is an emerging evidence base for a general myelosuppression in patients with chronic IHD, affecting both the functionality and the number of stem/progenitor cells in the BM. For example, in search for an explanation of HF-associated anemia, several groups reported a reduced proliferation and differentiation capacity of BM-derived progenitor cells in vitro in ischemic but not in dilated cardiomyopathy [11-14]. Jakob et al. demonstrated the in vivo clinical impact of this functional deficit: progenitor cells obtained from patients with ischemic cardiomyopathy showed a reduced capacity to promote cardiac function and neovascularization after myocardial injection in a hindlimb ischemia mice model when compared to progenitor cells acquired from healthy subjects [15]. Also in patients with isolated coronary artery disease (CAD), and thus without concomitant HF, intrinsic impairment of hematopoietic stem cell differentiation was observed [16].

Taken together, results from clinical trials suggest that the $\mathrm{BM}$ compartment in ischemic $\mathrm{HF}$, and also in isolated CAD, might be dysfunctional. So far, the extent and severity of BM suppression have not been compared between these various underlying etiologies. We hypothesize that the extent of BM dysfunction will be more prominent in the presence of $\mathrm{HF}$, since the existence of low-grade systemic inflammation in the HF syndrome may aggravate myelosuppression [17, 18].

In this regard, we performed a systematic evaluation of progenitor cell numbers and functionality in a broad range of cardiovascular diseases: $\mathrm{CAD}, \mathrm{HF}$ due to ischemic cardiomyopathy, HF due to non-ischemic cardiomyopathy, and compared this with a healthy control group. Moreover, inflammation was explored as a potential mechanism of BM dysfunction.

\section{Methods}

Detailed methods are available in online supplementary material.

\section{Study Population}

Thirty-two patients undergoing elective cardiac surgery via median sternotomy (coronary artery bypass grafting, mitral valve surgery, or implantation of ventricular assist device) were stratified into groups according to the presence of significant IHD and/or HF. Hence, there was a first group of patients with IHD without HF (i.e., CAD group), a second group with HF due to IHD (i.e., ischemic cardiomyopathy or ICM group), and a third group with $\mathrm{HF}$ due to other reasons than IHD (i.e., non-ischemic HF group). Eleven subjects without significant past history of cardiovascular disease served as controls. The study complied with the Helsinki Declaration and was approved by the local ethics committee, and written informed consent was obtained from all subjects.

\section{Sampling and Isolation of BM- and PB-MNC}

In patients, $\mathrm{BM}(2 \times 10 \mathrm{ml})$ was aspirated by sternal puncture under general anesthesia; arterial peripheral blood (PB, $12 \mathrm{ml})$ was sampled simultaneously via arterial catheter. In control subjects, $\mathrm{BM}$ was aspirated at the time of bone prelevation of the iliac crest prior to jaw reconstruction; venous $\mathrm{PB}$ was sampled by venipuncture. BM- and PB-MNC were isolated by density gradient centrifugation immediately after sampling. 


\section{Clinical and Biochemical Assessment}

All patients underwent coronary angiography and standard transthoracic echocardiography pre-operatively to determine the complexity of IHD (Syntax score) and LVEF, respectively. The Syntax score, an angiographic tool to quantify the complexity of coronary artery lesions, was calculated using the online Syntax score calculator [19]. Physical capacity was evaluated by the 6-min walk test. The Framingham risk score representing the 10-year risk for cardiovascular disease was calculated for each patient [20]. CRP, hemoglobin, creatinine, leukocyte, total cholesterol, HDL, and LDL circulating levels were measured pre-operatively.

\section{Assessment of BM Purity}

The percentage of bone matrix vesicle-bound bone alkaline phosphatase (ALP) of total ALP activity, as a marker for the purity of BM aspirates, was quantified in BM serum as described previously [21]. BM aspirates in which the percentage of bone matrix vesicle-bound ALP was less than $15 \%$ were excluded from the study for quality reasons.

\section{Transwell Migration Assay of BM-MNC}

In vitro migratory capacity of BM-MNC towards stromal cellderived factor- $1 \alpha$ (SDF- $1 \alpha, 100 \mathrm{ng} / \mathrm{ml}, \mathrm{R} \& D$ Systems Europe Ltd., UK) and vascular endothelial growth factor (VEGF, $50 \mathrm{ng} / \mathrm{ml}, \mathrm{R} \& \mathrm{D}$ Systems Europe Ltd.) was evaluated after 4 h using a modified Boyden chamber.

\section{Colony-Forming Unit Assays}

Isolated BM-MNC were aliquoted in duplicate in Methocult H4535 Enriched without EPO medium (Stem Cell Technologies, France) for induction of differentiation into the myeloid lineage (granulocyte/macrophage colonyforming units, GM-CFU), as well as in Methocult H4434 Classic medium (Stem Cell Technologies) for induction of differentiation into the erythroid lineage (erythroid burstforming units, BFU-E). After incubation for 14 days, the number of GM-CFU and BFU-E was counted.

\section{Paracrine Activity of BM-MNC}

Isolated BM-MNC were seeded on gelatin-coated 24-well plates $\left(5 \times 10^{6}\right.$ cells/well). After incubation for $24 \mathrm{~h}$, supernatant was collected, in which VEGF levels were measured using a human VEGF ELISA kit (R\&D Systems Europe Ltd.).
Flow Cytometry for Hematopoietic and Endothelial Progenitor Cell Enumeration

Multiparametric flow cytometry was applied for the assessment of the cellular composition of the BM-MNC fraction, as well as for the specific enumeration of hematopoietic (HPC, $\mathrm{CD} 5^{\mathrm{dim}} \mathrm{CD} 34^{+} \mathrm{SSC}^{\text {low }}$, ISHAGE gating strategy [22]) and endothelial (EPC, CD45 ${ }^{\mathrm{dim}} \mathrm{CD} 34^{+} \mathrm{KDR}^{+}$, EPC-gating protocol standardized by our laboratory [23]) progenitor cells in both BM- and PB-MNC samples using APC-H7-labeled CD45 (BD), PE-Cy7-labeled CD34 (BD), and APC-labeled KDR (R\&D Systems) antibodies. HPC and EPC numbers were expressed as relative numbers among CD45+ events. Early and late apoptosis was assessed by 7-AAD (BD) and FITC-labeled Annexin V staining (BD).

\section{Inflammatory and Angiogenic Cytokine Levels}

Levels of the inflammatory cytokines tumor necrosis factor alpha $(\mathrm{TNF} \alpha)$, interferon gamma (IFN $\gamma)$, and interleukin 6 (IL-6) and the angiogenic cytokines VEGF and basic fibroblast growth factor (bFGF) were measured in PB and BM plasma using Meso Scale Discovery multiplex platforms (Meso Scale Diagnostics, Maryland, USA).

\section{Molecular Expression of Inflammatory and Angiogenic Receptors}

Expression of TNFR1 and TNFR2 (TNF $\alpha$ receptors), IFNGR1 (IFN $\gamma$ receptor 1), IL6-R (IL-6 receptor), CXCR4 (SDF-1 $\alpha$ receptor), and VEGFR2 (VEGF receptor 2 ) in BMMNC was normalized to GAPDH and calculated by real-time qPCR using the $\Delta \Delta \mathrm{CT}$ method.

\section{Statistical Analysis}

Results are expressed as median (interquartile range (IQR)). IBM SPSS Statistics version 22.0 was used for statistical analysis. Normality of the variables was tested with Shapiro-Wilk and by visual $Q-Q$ plot inspection. Logarithmic transformation of skewed data was done to obtain a normal distribution. Comparison between different groups was performed using ANOVA (normally distributed data), Kruskal-Wallis (skewed data), or chi-square test (nominal data), as appropriate. To evaluate the specific influence of either IHD or HF, a twoway ANOVA was performed for each parameter with IHD, $\mathrm{HF}$, and the interaction between IHD and HF as independent factors. All models were adjusted for age. When the interaction between IHD and HF was not significant, it was removed from the model. Post hoc pairwise comparison was obtained by Mann-Whitney $U$ and adjusted with the Bonferroni-Holm correction for multiple testing. Correlations were evaluated by 
the Pearson or Spearman test, as appropriate. A $p$ value of $<0.05$ was considered statistically significant.

\section{Results}

\section{Patient Characteristics}

After exclusion of impure BM aspirates, i.e., samples that were contaminated with PB $(n=12), 43$ subjects were included in the analysis. Figure 1 shows the stratification into groups based on the presence of IHD and/or HF.

Demographic and clinical characteristics of the different cohorts are depicted in Table 1. Groups were similar with respect to age and gender. Syntax scores were similar between the ICM and CAD groups, indicating a comparable IHD complexity. Patients with ICM showed significantly lower LVEF compared to patients with non-ischemic HF ( $p=0.015)$, but New York Heart Association (NYHA) class was not different.

Furthermore, three control subjects received ACE inhibitors, beta-blockers, and/or statins as treatment for arterial hypertension or hypercholesterolemia, respectively.

\section{Functional Analysis of BM-MNC}

Table 2 shows the results for the various functional tests that were performed in BM-MNC in the four groups. The results of the two-way ANOVA model with IHD and HF are shown separately in Table 3. For all parameters, the interaction between IHD and HF was not significant and could be removed from the model.

This analysis revealed a significant decrease in myeloid (GM-CFU; $p=0.009$ ) and erythroid (BFU-E; $p=0.033$ ) differentiation capacity of BM-MNC in the presence of IHD.

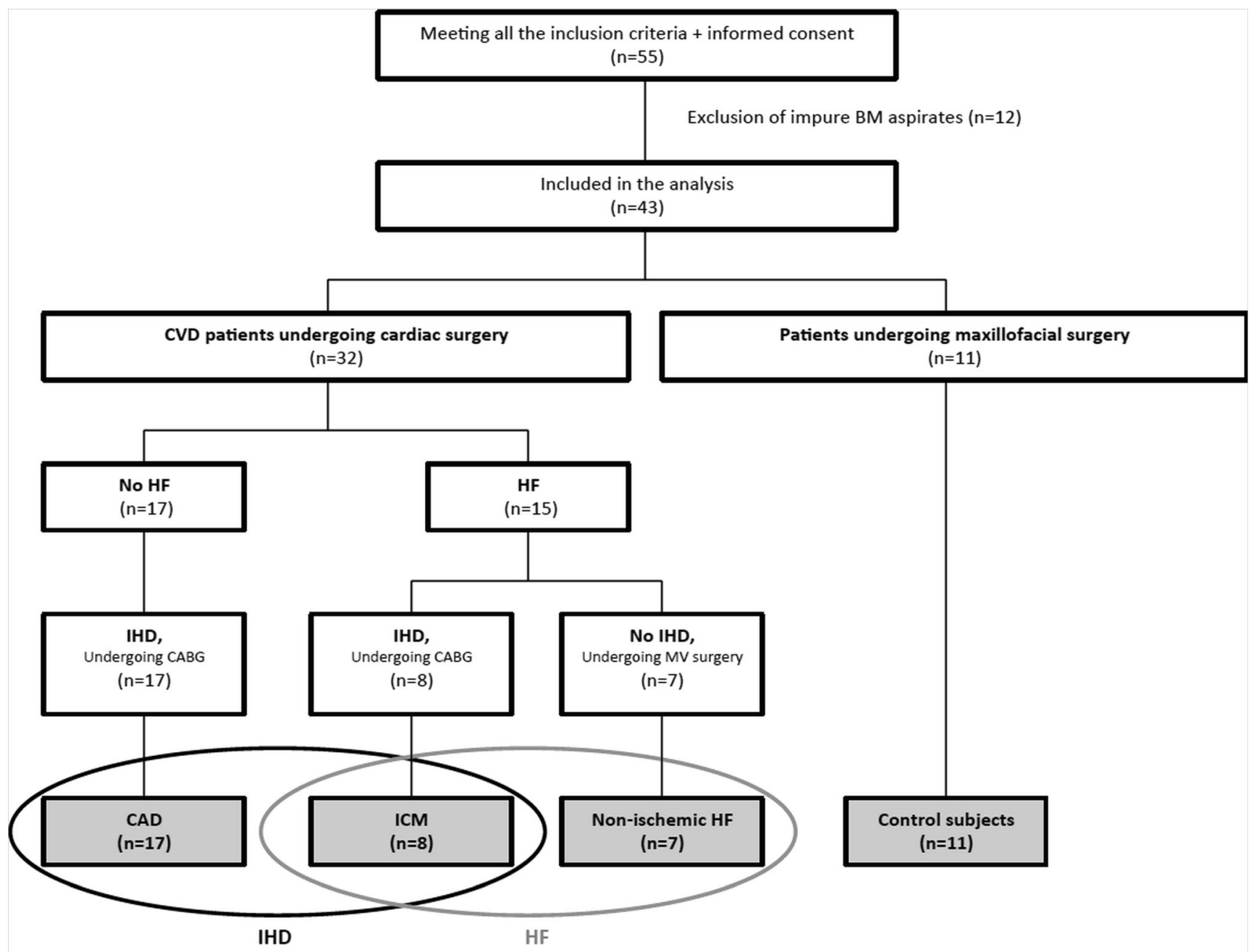

Fig. 1 Patient inclusion and stratification diagram. Abbreviations: $B M$, bone marrow; $C A D$, coronary artery disease; $C A B G$, coronary artery bypass grafting; $H F$, heart failure; $C V D$, cardiovascular disease; $I C M$, ischemic cardiomyopathy; $I H D$, ischemic heart disease; $M V$ surgery, mitral valve surgery 
Table 1 Demographic and clinical characteristics of the study population

\begin{tabular}{|c|c|c|c|c|c|}
\hline Group & $\mathrm{CAD}(n=17)$ & $\operatorname{ICM}(n=8)$ & Non-ischemic HF $(n=7)$ & Control subjects $(n=11)$ & $P$ value \\
\hline \multicolumn{6}{|l|}{ General } \\
\hline Age (years) & $65(60-72)$ & $60(42-72)$ & $59(52-64)$ & $55(40-66)$ & 0.055 \\
\hline Sex $(M)$ & 14 & 7 & 5 & 7 & 0.575 \\
\hline BMI $\left(\mathrm{kg} / \mathrm{m}^{2}\right)$ & $28(24-31)$ & $25(22-31)$ & $28(21-35)$ & $24(23-26)$ & 0.323 \\
\hline Systolic BP (mmHg) & $139(129-157)$ & $125(112-151)$ & $119(111-138)$ & N.A. & 0.177 \\
\hline \multicolumn{6}{|l|}{ Cardiac evaluation } \\
\hline IHD & + & + & - & - & $N . A$. \\
\hline $\mathrm{HF}$ & - & + & + & - & N.A. \\
\hline LVEF (\%) & $60(56-68)$ & $22(16-39)$ & $45(35-50)$ & N.A. & $<0.001$ \\
\hline NYHA I/II/III/IV & N.A. & $0 / 4 / 3 / 0$ & $0 / 3 / 3 / 2$ & N.A. & 0.352 \\
\hline$n$-vessel disease $(1 / 2 / 3)$ & $3 / 7 / 7$ & $2 / 2 / 4$ & $0 / 0 / 0$ & N.A. & $<0.001$ \\
\hline Syntax Score & $16(10-25)$ & $20(9-23)$ & N.A. & N.A. & 0.861 \\
\hline Framingham Score & $16(11-26)$ & $15(4-24)$ & $8(2-15)$ & N.A. & 0.191 \\
\hline 6-MWT (m) & $556(500-638)$ & $350(260-400)$ & $600(524-614)$ & N.A. & 0.081 \\
\hline \multicolumn{6}{|c|}{ Hematological and biochemical analysis } \\
\hline $\mathrm{Hb}(\mathrm{g} / \mathrm{dl})$ & $13.5(13.0-14.4)$ & $15.1(12.0-15.8)$ & $12.8(12.4-14.8)$ & $14.6(12.7-15.7)$ & 0.524 \\
\hline Leukocytes (10E9/1) & $6.2(5.5-7.6)$ & $8.4(7.1-9.4)$ & $8.6(7.7-9.5)$ & $7.0(5.5-8.4)$ & 0.073 \\
\hline Creatinine (mg/dl) & $0.9(0.8-1.1)$ & $1.1(0.9-1.3)$ & $0.9(0.8-1.1)$ & $0.8(0.7-1.0)$ & 0.029 \\
\hline eGFR (ml/min) & $84(71-94)$ & $75(67-82)$ & $82(73-98)$ & $94(89-103)$ & 0.149 \\
\hline $\mathrm{CRP}(\mathrm{mg} / \mathrm{l})$ & $1.5(1.5-2.3)$ & $5.7(1.5-19.2)$ & $3.4(1.5-10.0)$ & $1.5(1.5-4.0)$ & 0.096 \\
\hline $\mathrm{TG}(\mathrm{mg} / \mathrm{dl})$ & $114(88-210)$ & $118(88-262)$ & $114(77-140)$ & N.A. & 0.301 \\
\hline Total cholesterol (mg/dl) & $170(139-190)$ & $210(138-226)$ & $208(164-249)$ & N.A. & 0.470 \\
\hline HDL (mg/dl) & $44(30-55)$ & $37(35-45)$ & $52(43-61)$ & N.A. & 0.019 \\
\hline LDL (mg/dl) & $94(65-119)$ & $144(75-157)$ & $139(101-170)$ & N.A. & 0.249 \\
\hline \multicolumn{6}{|l|}{ Medication $(N)$} \\
\hline ACE inhibitors/ARB & 8 & 5 & 4 & 2 & 0.200 \\
\hline Beta-blockers & 11 & 4 & 6 & 1 & 0.006 \\
\hline Diuretics & 4 & 5 & 6 & 0 & 0.001 \\
\hline Statins & 16 & 5 & 1 & 2 & $<0.001$ \\
\hline \multicolumn{6}{|l|}{ Surgery $(N)$} \\
\hline CABG & 17 & 6 & 0 & 0 & $<0.001$ \\
\hline MV surgery & 0 & 1 & 7 & 0 & $<0.001$ \\
\hline VAD & 0 & 2 & 0 & 0 & 0.027 \\
\hline Maxillofacial & 0 & 0 & 0 & 11 & $<0.001$ \\
\hline
\end{tabular}

Data are expressed as median (IQR). $P$ values for comparison between groups by ANOVA, Kruskal-Wallis, or chi-square test, as appropriate $A C E$ angiotensin-converting enzyme, $A R B$ angiotensin II receptor blockers, $B P$ blood pressure, $B M I$ body mass index, $C A D$ coronary artery disease, $H F$ heart failure, $C A B G$ coronary artery bypass grafting, $C R P$ C-reactive protein, $e G F R$ estimated glomerular filtration rate, $H b$ hemoglobin, $H D L$ highdensity lipoprotein, $I C M$ ischemic cardiomyopathy, $I H D$ ischemic heart disease, $L D L$ low-density lipoprotein, $L V E F$ Left ventricular ejection fraction, $M V$ mitral valve, $N Y H A$ New York Heart Association class, $T G$ triglycerides, VAD ventricular assist device, 6-MWT 6-min walk test

This is illustrated in Fig. 2a, b. The presence of HF did not affect the differentiation capacity of BM-MNC; however, within HF patients, a significant positive correlation between GM-CFU numbers and LVEF was observed (Fig. 2c).

Additionally, whereas migratory capacity of BM-MNC did not differ between groups, it strongly correlated with IHD complexity, as assessed by the Syntax score
(Fig. 2d). VEGF production of isolated BM-MNC was measured in a subset of the study population (Table 2), but no differences in VEGF production after 24-h incubation were detected between groups.

For the total cohort, a significant negative relation between age and GM-CFU $(r=-0.416 ; p=0.014$; Fig. $2 \mathrm{e})$ and BFU-E $(r=-0.332 ; p=0.042$; Fig. 2f) was observed. 
Table 2 Functional/numerical assessment of MNC in BM and PB

\begin{tabular}{|c|c|c|c|c|}
\hline Parameter & CAD & $\mathrm{ICM}$ & Non-ischemic HF & Control subjects \\
\hline \multicolumn{5}{|l|}{ Bone marrow } \\
\hline Functional analysis & $N=17$ & $N=8$ & $N=7$ & $N=11$ \\
\hline Migration capacity (\%) BM-MNC & $31(20-38)$ & $26(18-31)$ & $29(26-35)$ & $32(23-39)$ \\
\hline GM-CFU $(N)$ & $42(21-55)$ & $43(33-69)$ & $68(37-114)$ & $73(55-82)$ \\
\hline \multirow[t]{2}{*}{ BFU-E $(N)$} & $29(18-36)$ & $31(27-42)$ & $25(17-61)$ & $40(36-55)$ \\
\hline & $N=13$ & $N=5$ & $N=2$ & $N=4$ \\
\hline VEGF (pg/ml) & $138(91-191)$ & $132(89-380)$ & 121 (97-N.A.) & $175(119-227)$ \\
\hline Numerical analysis & $N=17$ & $N=8$ & $N=7$ & $N=11$ \\
\hline BM HPC (/10 $0^{6}$ CD5 $5^{+}$BM-MNC) & $12117(8329-16465)$ & 14309 (9166-16839) & $1196(5800-22089)$ & $16703(12076-20914)$ \\
\hline BM EPC (/10 $\left.0^{6} \mathrm{CD} 45^{+} \mathrm{BM}-\mathrm{MNC}\right)$ & $310(118-529)$ & $255(64-796)$ & $136(38-304)$ & $242(80-508)$ \\
\hline Early apoptosis (\%) BM-MNC & $0.33(0.16-0.60)$ & $0.24(0.06-0.32)$ & $0.65(0.33-0.92)$ & $0.42(0.26-0.59)$ \\
\hline Late apoptosis (\%) BM-MNC & $0.25(0.11-0.70)$ & $0.09(0.04-0.30)$ & $0.74(0.31-1.05)$ & $0.52(0.14-0.85)$ \\
\hline \multicolumn{5}{|l|}{ Peripheral blood } \\
\hline Numerical analysis & $N=13$ & $N=5$ & $N=7$ & $N=11$ \\
\hline PB HPC $\left(/ 10^{6}\right.$ CD45 $5^{+}$PB-MNC) & $863(534-1153)$ & $2089(889-2453)$ & $1056(646-1261)$ & $672(589-798)$ \\
\hline PB EPC ( $\left./ 10^{6} \mathrm{CD}^{2} 5^{+} \mathrm{PB}-\mathrm{MNC}\right)$ & $76(40-112)$ & $299(68-464)$ & $26(11-74)$ & $29(17-39)$ \\
\hline Early apoptosis (\%) PB-MNC & $0.23(0.15-0.32)$ & $0.11(0.08-0.29)$ & $0.16(0.07-0.38)$ & $0.08(0.04-0.11)$ \\
\hline Late apoptosis (\%) PB-MNC & $0(0-0.01)$ & $0(0-0.02)$ & $0.01(0-0.01)$ & $0.01(0-0.01)$ \\
\hline
\end{tabular}

Data are expressed as median (IQR). Abbreviations as in Table 1

$B F U-E$ erythroid burst-forming units, $B M$ bone marrow, $E P C$ endothelial progenitor cells, $G M-C F U$ granulocyte-macrophage colony-forming units, $H P C$ hematopoietic progenitor cells, $M N C$ mononuclear cells, $P B$ peripheral blood, $V E G F$ vascular endothelial growth factor

Table 3 Parameter estimates resulting from ANOVA analysis for numerical and functional assessments in BM and PB

\begin{tabular}{|c|c|c|c|c|c|c|c|}
\hline \multirow[t]{2}{*}{ Parameter } & \multicolumn{3}{|l|}{ IHD } & \multicolumn{3}{|l|}{$\mathrm{HF}$} & \multirow{2}{*}{$\begin{array}{l}\text { Model } \\
P \text { value }\end{array}$} \\
\hline & Beta coefficient & SE & $P$ value & Beta coefficient & SE & $P$ value & \\
\hline \multicolumn{8}{|l|}{ Bone marrow } \\
\hline \multicolumn{8}{|l|}{ Functional analysis } \\
\hline Migratory capacity (\%) BM-MNC & -1.585 & 3.143 & 0.617 & -3.214 & 3.030 & 0.296 & 0.521 \\
\hline GM-CFU $(N)$ & -25.076 & 8.930 & 0.009 & 3.550 & 8.768 & 0.688 & 0.012 \\
\hline BFU-E $(N)$ & -10.106 & 4.553 & 0.033 & -0.302 & 4.597 & 0.948 & 0.083 \\
\hline \multicolumn{8}{|l|}{ Numerical analysis } \\
\hline BM HPC (/10 $0^{6}$ CD45+ BM-MNC) & -1175.230 & 1897.721 & 0.540 & -1631.138 & 1855.751 & 0.385 & 0.200 \\
\hline Log BM EPC (/10 $0^{6}$ CD45+ BM-MNC) & 0.130 & 0.165 & 0.436 & -0.181 & 0.161 & 0.268 & 0.317 \\
\hline Log early apoptosis (\%) BM-MNC & -0.294 & 0.146 & 0.051 & -0.097 & 0.142 & 0.500 & 0.133 \\
\hline Log late apoptosis (\%) BM-MNC & -0.333 & 0.154 & 0.037 & -0.144 & 0.151 & 0.346 & 0.059 \\
\hline \multicolumn{8}{|l|}{ Peripheral blood } \\
\hline \multicolumn{8}{|l|}{ Numerical analysis } \\
\hline Log PB HPC (/10 CD45+ BM-MNC) & 0.147 & 0.075 & 0.058 & 0.187 & 0.068 & 0.010 & 0.029 \\
\hline Log PB EPC (/10 CD45+ BM-MNC) & 0.612 & 0.133 & $<0.001$ & 0.255 & 0.121 & 0.042 & $<0.001$ \\
\hline Log early apoptosis (\%) PB-MNC & 0.201 & 0.136 & 0.149 & 0.080 & 0.123 & 0.522 & 0.234 \\
\hline Log late apoptosis (\%) PB-MNC & -0.018 & 0.223 & 0.936 & 0.213 & 0.203 & 0.304 & 0.547 \\
\hline
\end{tabular}

Abbreviations as in Tables 1 and 2. The regression model tested IHD and HF as independent factors (adjusted for age) for each parameter. All models were adjusted for age

SE standard error 

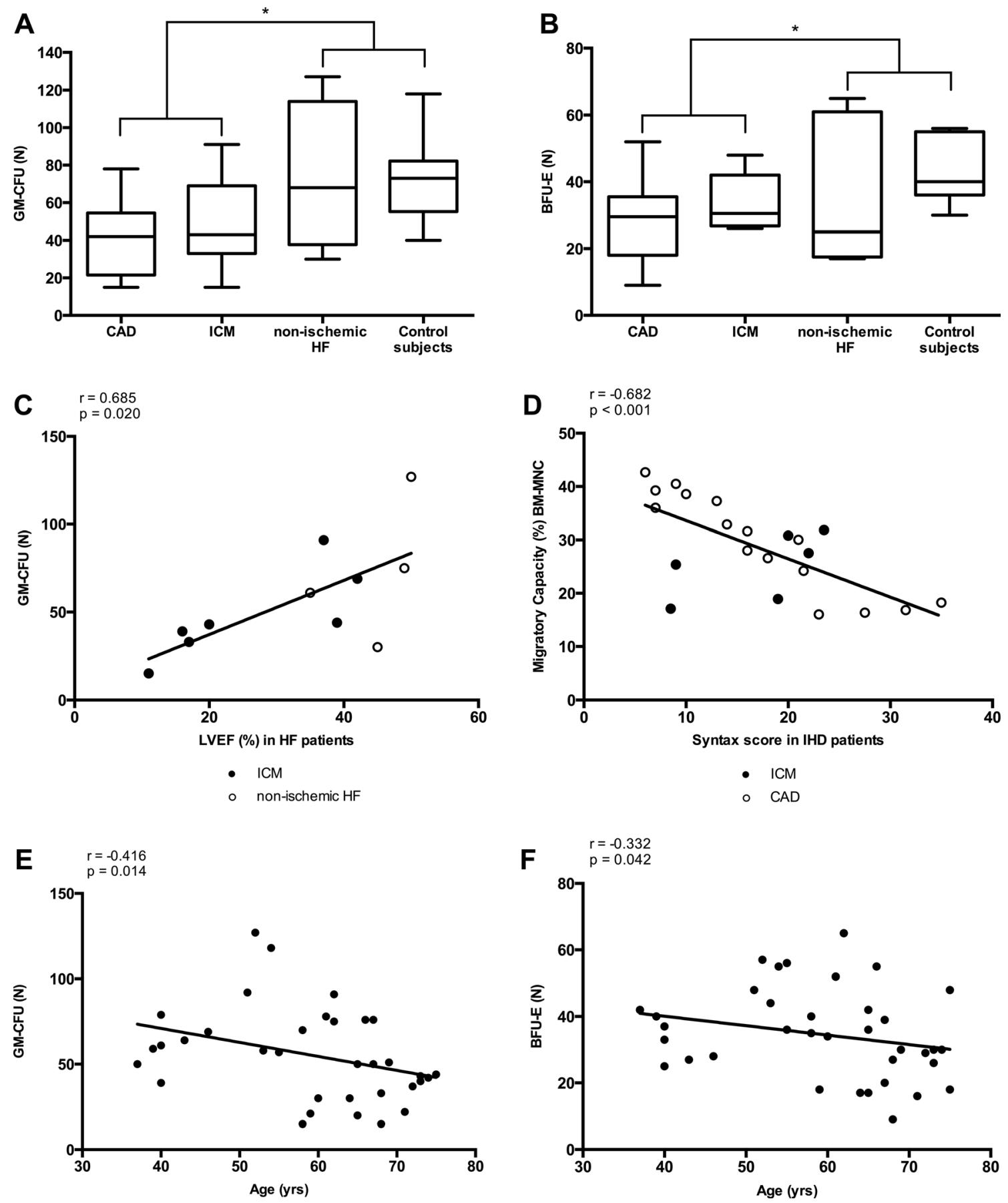

Fig. 2 Functional assessment of BM-MNC of patients with IHD and HF. a In vitro myeloid (GM-CFU numbers) and b erythroid (BFU-E numbers) differentiation capacities of BM-MNC were decreased in subjects with IHD (i.e., CAD and ICM group, $n=17+8$ ) compared to subjects without IHD (i.e., non-ischemic HF group and control subjects,

To exclude the possibility that the observed differences in myeloid and erythroid differentiation capacity are related to differences in cellular composition of the BM-MNC sample, a multiparametric flow cytometric analysis of BM-MNC was performed. In exception of higher monocyte percentages in

$n=7+11)$. $\mathbf{c}$ In HF patients, GM-CFU numbers decrease with decreasing LVEF. $\mathbf{d}$ In IHD patients, migratory capacity of BM-MNC decreases with increasing IHD complexity, i.e., Syntax score. ${ }^{*} p<0.05$ (ANOVA). e For the total cohort, age was negatively related with GM-CFU numbers and $\mathbf{f}$ BFU-E numbers

IHD patients, BM from subjects with and subjects without IHD were comparable in cellular composition, including the ratio of lymphoblasts versus $\mathrm{CD} 117+$ myeloblasts $(p=0.347)$ or versus $\mathrm{CD} 33+$ myeloblasts $(p=0.347)$ present in the BMMNC pool (Supplementary Table 1). 


\section{Numerical Analysis of Progenitor Cells in BM and PB}

In the BM compartment, HPC and EPC numbers were comparable between the four groups (Table 2) and were not influenced by the presence of IHD and/or HF (Table 3). The percentage of BM-MNC in early and late apoptosis was significantly lower in subjects with IHD compared to subjects without IHD (Table 3).

In PB, EPC numbers were significantly elevated in the presence of IHD ( $p<0.001$; Fig. 3a), as well as in the presence of HF ( $p=0.042$, Fig. 3a). Moreover, an inverse correlation between circulating EPC numbers and LVEF was found in all patients with HF (Fig. 3b).

The presence of HF also affected circulating HPC numbers with higher numbers of HPC in subjects with HF compared to subjects without HF ( $p=0.010$, Table 3 and Fig. $3 c)$.

Early and late apoptotic BM-MNC percentages were more prominent in subjects without IHD than with IHD $(p=0.051$ and $p=0.037$; Table 3 ). Yet, the degree of early and late
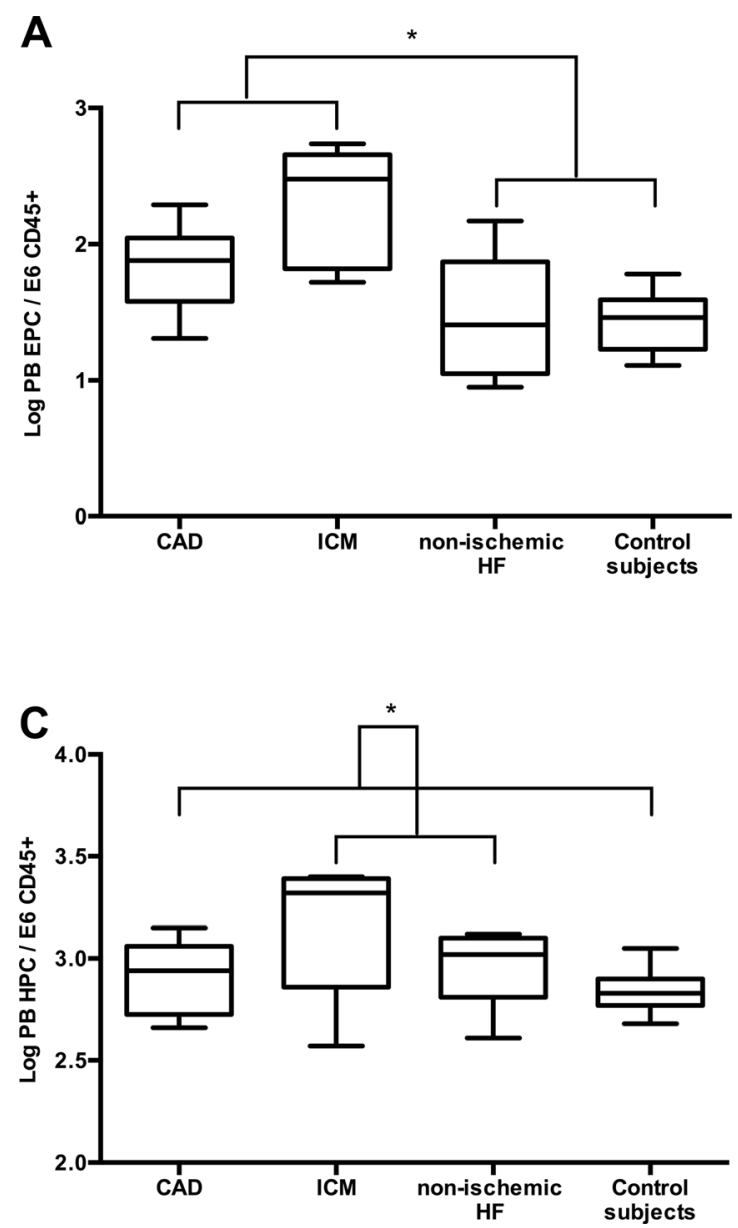

Fig. 3 Enumeration of circulating hematopoietic (HPC) and endothelial $(E P C)$ progenitor cells in patients with IHD and HF. a Circulating EPC numbers were increased in subjects with IHD (i.e., CAD and ICM group, $n=13+5$ ) compared to subjects without IHD (i.e., non-ischemic HF group and control subjects, $n=7+11)$. b Circulating EPC negatively apoptosis of freshly isolated MNC both in BM and PB was overall minimal $( \pm<1 \%)$.

\section{Inflammatory and Angiogenic Cytokine Profile in BM and PB}

To explore the mechanisms responsible for the aforementioned functional and numerical differences, levels of inflammatory cytokines and pro-angiogenic growth factors were studied in a subpopulation of 25 subjects.

In this entire subpopulation, levels of inflammatory cytokines were lower in $\mathrm{BM}$ compared to $\mathrm{PB}$ (IFN $\gamma, \mathrm{TNF} \alpha$, and IL-6), whereas levels of angiogenic growth factors (VEGF and $\mathrm{bFGF}$ ) were higher in $\mathrm{BM}$ compared to $\mathrm{PB}$ (Supplementary Table 2).

The comparison between the four study groups is shown in Table 4. Only IL-6 levels were significantly altered: pairwise comparison showed a significant elevation of IL-6 in PB $(p=0.015)$, as well as in $\mathrm{BM}(p=0.013)$ in patients with

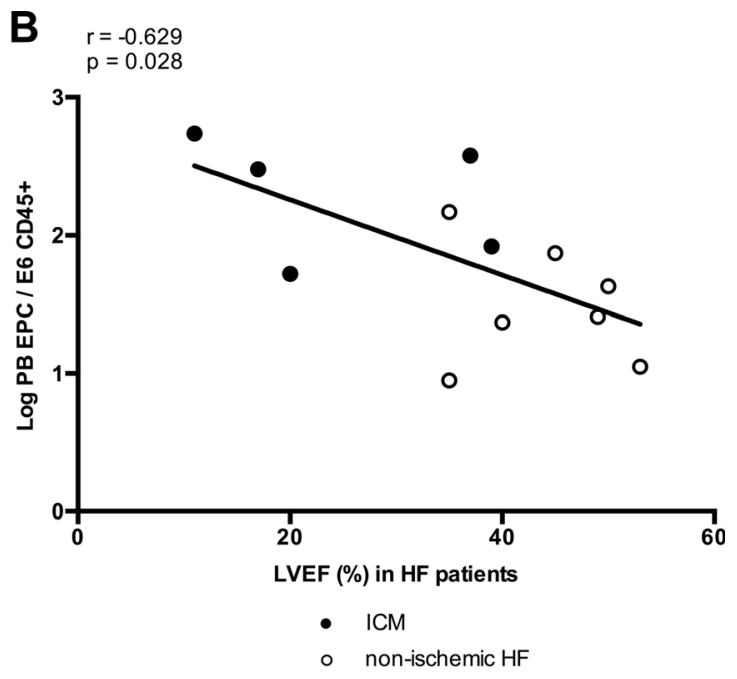

correlated with LVEF in patients with HF. c Circulating HPC numbers were elevated in subjects with HF (ICM and non-ischemic HF group, $n=5+7$ ) compared to subjects without HF (CAD group and control subjects, $n=13+11) .{ }^{*} p<0.05$ (ANOVA) 
Table 4 Cytokine levels in BM and PB

\begin{tabular}{|c|c|c|c|c|c|}
\hline & CAD & $\mathrm{ICM}$ & Non-ischemic HF & Control subjects & $P$ value \\
\hline Bone marrow & $N=6$ & $N=6$ & $N=5$ & $N=8$ & \\
\hline $\mathrm{BM} \operatorname{IFN} \gamma(\mathrm{pg} / \mathrm{ml})$ & $4.39(3.25-4.81)$ & $3.75(0.48-5.64)$ & $4.25(2.42-9.72)$ & $1.07(0.20-3.20)$ & 0.065 \\
\hline $\mathrm{BM} \mathrm{TNF} \alpha(\mathrm{pg} / \mathrm{ml})$ & $1.94(1.59-2.64)$ & $1.61(1.10-2.89)$ & $1.80(1.53-2.07)$ & $1.29(1.05-1.63)$ & 0.185 \\
\hline BM IL-6 (pg/ml) & $0.34(0.05-0.65)$ & $0.88(0.52-1.53)$ & $0.84(0.16-1.40)$ & $0.18(0.05-0.48)$ & 0.049 \\
\hline BM VEGF (pg/ml) & 66.79 (48.28-91.17) & 79.69 (60.98-228.84) & $59.67(27.02-97.54)$ & $229.06(70.85-282.83)$ & 0.081 \\
\hline BM bFGF (pg/ml) & $58.05(48.32-304.51)$ & $122.13(97.53-181.18)$ & $300.82(137.74-548.76)$ & $13676(81.78-334.45)$ & 0.141 \\
\hline Peripheral blood & $N=6$ & $N=6$ & $N=5$ & $N=8$ & \\
\hline PB IFN $\gamma(\mathrm{pg} / \mathrm{ml})$ & $4.35(3.25-5.82)$ & $4.70(1.83-6.75)$ & $4.97(3.50-12.63)$ & $4.34(2.44-4.66)$ & 0.509 \\
\hline PB TNF $\alpha(\mathrm{pg} / \mathrm{ml})$ & $2.22(1.58-2.65)$ & $2.03(1.05-3.09)$ & $2.04(1.76-2.48)$ & $1.74(1.32-2.00)$ & 0.733 \\
\hline PB IL-6 (pg/ml) & $0.22(0.05-0.59)$ & $1.01(0.59-1.97)$ & $1.06(0.53-1.25)$ & $0.37(0.11-0.51)$ & 0.007 \\
\hline PB VEGF (pg/ml) & $16.52(14.76-66.51)$ & $30.78(10.47-132.84)$ & $31.45(17.96-80.66)$ & 83.34 (23.59-101.65) & 0.523 \\
\hline PB bFGF (pg/ml) & $2.45(2.22-5.39)$ & $3.09(1.59-4.74)$ & $2.88(2.83-4.39)$ & $2.51(0.77-19.16)$ & 0.971 \\
\hline
\end{tabular}

Abbreviations as in Tables 1 and 2. Data are expressed as median (IQR). $P$ values for comparison between groups by Kruskal-Wallis $b F G F$ basic fibroblast growth factor, $I F N \gamma$ interferon gamma, $I L-6$ interleukin $6, T N F \alpha$ tumor necrosis factor alpha

ICM compared to control subjects. The increases in IL6 levels in $\mathrm{BM}$ and $\mathrm{PB}$ in patients with non-ischemic $\mathrm{HF}$ were not significant (PB IL-6, $p=0.059$ and BM IL-6, $p=0.113$ ); however, both BM and PB IL-6 concentrations correlated negatively with LVEF (BM IL-6, $r=-0.556, p=0.025$; PB IL-6, $r=-0.708, p=0.002$ ) and 6-MWT (BM IL-6, $-0.886, p=0.019$; PB IL-6, $r=-0.886, p=0.019)$. Moreover, $\mathrm{TNF} \alpha$ and IFN $\gamma$ levels in $\mathrm{BM}$ were increased in patients with CAD compared to control subjects, but this was not significant.

Neither IL-6, TNF $\alpha$, IFN $\gamma$, nor VEGF and bFGF levels in $\mathrm{BM}$ and $\mathrm{PB}$ correlated with any functional parameter or progenitor cell number in the study population.

\section{Cytokine Receptor Expression on BM-MNC}

Expression of cytokine receptors (TNFR1 and 2, IFNGR1, IL6R) and receptors involved in the mobilization of BM-MNC into the circulation (VEGFR2, CXCR4) were analyzed to evaluate the sensitivity of the BM-MNC to the aforementioned cytokines and to gain more insight in the leading factor for mobilization.

Expression levels of these receptors on BM-MNC did not show any significant up- or downregulation between groups and thus appeared to be unaffected by either the presence of IHD and/or HF (Supplementary Table 3).

An increase in TNF $\alpha$ levels in $\mathrm{BM}$ was associated with an upregulation of TNFR2 expression (BM, $r=$ $0.536, p=0.007$ ) in the study population. In turn, TNFR2 expression was negatively related to both GMCFU $(r=-0.428, p=0.015)$ and BFU-E $(r=-0.629, p=$ $0.005)$ numbers. Moreover, the ratio of TNFR1 to
TNFR2 expression on BM-MNC, which gives an indication for cell survival (low ratio) or apoptosis (high ratio), did not differ between groups. In patients with $\mathrm{HF}$, no increase in TNF $\alpha$ was observed, but a negative correlation with LVEF and TNFR1 expression on BMMNC $(r=-0.642, p=0.010)$ was documented. Circulating EPC numbers were correlated with CXCR4 expression on BM-MNC $(r=0.364, p=0.034)$.

\section{Discussion}

The present study is the first to provide a comprehensive analysis of the numbers and function of BM-derived progenitor cells in the setting of IHD and HF. Several interesting findings emerge from this study:

1. Both myeloid and erythroid differentiation capacities of BM-MNC are impaired in the presence of IHD. Moreover, BM-MNC show attenuated migratory capacity with increasing complexity of angiographic lesions.

2. We did not observe a depletion of progenitor cells in the BM compartment, neither in IHD nor in HF. These data suggest that the impairment of progenitor cells in the BM in IHD is restricted to a functional limitation and not to numerical values.

3. The decline in functionality of BM-MNC in IHD is not driven by low-grade systemic inflammation.

4. EPC mobilization from the BM into the circulation in IHD patients appears to involve CXCR4-mediated signaling. 


\section{Functional Impairment of BM-Derived Progenitor Cells in IHD}

Several studies previously reported reduced in vitro GM-CFU and BFU-E capacity of isolated BM-MNC in the setting of ICM (LVEF $<45-40 \%$, NYHA II-IV) compared to healthy subjects $[11,12,14]$. Liguori et al. also observed this functional impairment in patients with CAD without the clinical presence of HF (LVEF $>45 \%$, NYHA I) [16]. In this study, we confirmed the reduced myeloid/erythroid differentiation capacity of BM-MNC in CAD but distinguished the effect of IHD versus HF. Surprisingly, we identified only IHD, but not HF, as an important determinant of BM-MNC differentiation capacity. This finding is in line with the work of Kissel et al. who observed lower GM-CFU numbers in ICM patients (previous MI, LVEF $32 \pm 11 \%$ ) but not in age-matched patients with dilated cardiomyopathy (LVEF $27 \pm 9 \%$ ) [12].

Heeschen et al. also documented a reduced migratory response towards SDF- $1 \alpha$ and VEGF of BM-MNC in 18 patients with ICM (previous MI, LVEF $38 \pm 11 \%$ ) [14]. In our study, however, migratory capacity of BM-MNC was not influenced by the presence of IHD nor by HF. This discrepancy could be explained by the differences in study population: the ICM patients in the study of Heeschen et al. were $17 \%$ diabetic and compared to significantly younger healthy subjects [14]. Both diabetes and age are known influencing determinants of the progenitor cell function (see infra) [24, 25]. Therefore, we assigned diabetes as exclusion criterion in our study and corrected for the confounding age effect in our analyses.

In the present study, we demonstrated a significant inverse relation between the migratory capacity of BM-MNC and the complexity of IHD, as assessed by the Syntax score. The observation that severity of IHD is an important determinant of progenitor cell function has been suggested earlier by Bozdag-Turan et al.: CD34+/CD45+ and CD133+/CD45+ progenitor cells isolated from peripheral blood showed a reduced erythroid-myeloid differentiation capacity and migratory response towards VEGF/SDF- $1 \alpha$ in patients with IHD, which all decreased further with increasing number of diseased coronary arteries [26].

In conclusion, this study extends previous observations and shows that, in the setting of IHD, myeloid and erythroid differentiation capacities of progenitor cells are already impaired when still residing in the BM compartment. This impairment in functionality worsens further with increasing complexity of CAD but is not observed in non-ischemic HF. Our findings identify IHD rather that the HF syndrome itself as primum movens for the observed functional impairment of BM cells. We acknowledge that these results do not support our initial hypothesis: since HF is regarded as a multi-system disorder that also accounts for several peripheral abnormalities such as endothelial dysfunction, skeletal muscle changes, and ventilatory inefficiency [27], we also anticipated an associated $\mathrm{BM}$ dysfunction. Yet, our results suggest otherwise and are in accordance with the findings from Kissel et al. [12] and Liguori et al. [16], as discussed above. In this regard, our focus in the quest to reveal the underlying mechanisms for this functional BM deficit in this patient group should also shift towards ischemia-induced pathways.

\section{Increased EPC Recruitment into the Circulation in IHD}

The fact that we did not observe a depletion of HPC and EPC in the BM compartment, neither in IHD nor in HF, is in line with the findings from Kissel et al. [12] and Heeschen et al. [14], who did not find any differences in ICM compared to healthy subjects either. However, while we used the ISHAGE characterization $\left(\mathrm{CD} 45^{\mathrm{dim}} \mathrm{CD} 34^{+} \mathrm{SSC}^{\text {low }}\right.$ ) for HPC enumeration in $\mathrm{BM}$, both groups applied $\mathrm{CD} 34^{+} \mathrm{CD} 45^{+} \mathrm{BM}-\mathrm{MNC}$ as HPC phenotype characterization and $\mathrm{CD} 34^{+} \mathrm{CD} 133^{+} \mathrm{BM}-$ $\mathrm{MNC}$ for immature HPC. This is in contrast with the findings from Liguori et al. [16], who reported reduced HPC numbers in the $\mathrm{BM}$ of patients with CAD.

Whereas we observed a functional impairment of BMderived progenitor cells in IHD, we also observed elevated circulating EPC numbers in these patients. This finding implies an active, possibly compensatory, recruitment of EPC from the BM into the circulation in the setting of ischemia, in order to obtain local neovascularization of ischemic tissue. In our study, higher numbers of circulating EPC correlated with higher CXCR4 expression on BM-MNC, suggesting that $\mathrm{EPC}$ recruitment into the circulation is mediated, at least partly, by SDF- $1 \alpha$.

In literature, conflicting results in circulating EPC numbers in the setting of IHD and HF have been published. Vasa et al. [28] showed a decrease in $\mathrm{CD} 34^{+} \mathrm{KDR}^{+}$cells in the circulation of CAD patients (stable CAD, acute coronary syndrome, or MI) compared to healthy subjects, while Valgimigli et al. [29] demonstrated increased $\mathrm{CD} 34^{+} \mathrm{CD} 133^{+} \mathrm{KDR}^{+}$numbers in $\mathrm{PB}$ of patients with congestive HF (from different etiologies), especially in the early phase of HF (NYHA I). These data emphasize that not only the heterogeneity in EPC phenotypes but also the differences in severity of HF can lead to variations in study outcome.

\section{Underlying Mechanisms for BM-Derived Progenitor Cell Dysfunction in IHD}

Multiple mechanisms could underlie the observed dysfunction of BM-derived progenitor cells in IHD. Identification of these targets could help to restore progenitor cell functions and improve outcome after autologous stem cell therapy.

Since TNF $\alpha$ has been identified as a cell-extrinsic suppressor of normal HPC activity in vivo [18], we explored increased inflammation as a potential trigger of BM dysfunction. 
Interestingly, the inflammatory cytokine levels (TNF $\alpha, \operatorname{IFN} \gamma$, and IL-6) were lower in BM compared to PB, suggesting that $\mathrm{BM}$ can be regarded as a separate compartment and is protected from systemic increases in circulating cytokines. As expected, IL-6 levels were increased in both circulation and BM of patients with ICM; however, these IL-6 elevations were not statistically significant in patients with non-ischemic HF, which might be attributed to a less depressed LVEF in this patient cohort.

Neither the levels of TNF $\alpha$ and IFN $\gamma$ in the BM compartment nor their systemic levels differed between groups. A possible hypothesis for the lack of these differences in inflammatory markers could potentially be ascribed to the inherent immunosuppressive nature of the BM-derived progenitor cells. Not only MSC but also early myeloid progenitor cells have recently been identified as immunosuppressors by the induction of $\mathrm{T}$ cell proliferation and regulatory $\mathrm{T}$ cell development in vitro [30]. The underlying mechanisms for these immunomodulatory properties are not yet fully elucidated, but MSC-mediated immunosuppression appeared to be even induced by pro-inflammatory cytokines through the role play of chemokines and NO, suggesting a compensatory mechanism [31].

TNFR2 is known to act as life receptor stimulating proliferation and cell survival, particularly in lymphocytes [32]. In contrast, we documented a negative relation between TNFR2 and both myeloid and erythroid differentiation capacity of BM-MNC. Yet, data by Schling et al. clearly demonstrate that there is no general principle for the regulated expression of $\mathrm{TNF} \alpha$ and its receptors during the conversion of precursors into a more mature cell phenotype [33]. In this regard, experimental studies with primary cultures from WT and TNFR2 $-/-$ mice have demonstrated accelerated differentiation from bone marrow precursor cells into dendritic cells and osteoclasts $[34,35]$.

Based on these findings, as well as on the missing correlations between the inflammatory markers and the progenitor cell functionality in our study population, it is rather unlikely that inflammation is a major underlying mechanism for the observed BM dysfunction in IHD. In hematological disorders, immunosuppressive therapy has even been shown to beneficially affect the BM function. For example, in patients with aplastic anemia, immunosuppressive therapy with antithymocyte globulin and cyclosporine A results in hematologic recovery of the BM, despite the initial defect in progenitor cell numbers [36]. Hence, we should not ignore the suppressing role of inflammation on the progenitor cell function, but our data suggest that in the setting of IHD other more determining mechanisms are at play, whether or not stimulated by inflammation.

Since the intrinsic cellular dysfunction in BM was not observed in the setting of HF, future experiments could focus on ischemia-related pathways as underlying mechanism for BM dysfunction. For example, diabetes-induced microangiopathy in BM is responsible for a reduced perfusion and nutrition, as well as increased oxidative stress and reduced nitric oxide bioavailability, thereby hampering the progenitor cell function and HPC/EPC mobilization in ischemia models in vivo [37]. The suppressing effect of microvascular disease on BM function could be further extrapolated to IHD, although, the extent of microvascular disease in the BM niche in IHD has to be further explored.

Accelerated senescence of BM-derived progenitor cells could also be a potential underlying mechanism since IHD has been associated with telomere shortening of BM-MNC [38].

\section{Implications for Autologous Stem Cell Therapy}

Impaired progenitor cell function compromises the outcome of autologous stem cell therapy. The TOPCARE-CHD study demonstrated the clinical relevance of this impairment: administration of progenitor cells with a reduced function in vitro is inferior to administration of BM-MNC with a preserved function on patient survival and LVEF improvement [8]. A detailed analysis performed by Cogle et al. on the stem cell product that was administered in patients with acute and chronic IHD and left ventricular dysfunction showed a decrement in LVEF with decreasing percentages of CD34+ of the administered BM-MNC [13]. Based on our results, it appears that the poor outcome of autologous stem cell therapy in IHD might be a consequence of their reduced ability to differentiate.

In order to identify suitable therapeutic targets to improve efficacy of autologous stem cell therapy, we should first collect more insight in the underlying mechanisms of this observed progenitor cell dysfunction in IHD patients. Secondly, these specific pathways could be targeted to reverse the functional outcome. In this regard, modulation of cellular microRNA is particularly promising and proven successful in augmenting the progenitor cell function by means of paracrine activity, cell survival, and cardiomyogenic differentiation capacity, with direct beneficial impact on cardiac and/or vascular regeneration [39].

\section{Additional Determinants of the Progenitor Cell Function}

Not only the presence of IHD but also specific demographic characteristics of the study population are well-known relevant determinants on the BM-derived progenitor cell function.

For example, evidence has been collected for agedependent deterioration of the proliferative capacity of BMderived progenitor cells and in general the hematopoietic compartment [24]. In healthy subjects and/or CAD patients, age has been associated with impaired in vivo neovascularization, in vitro migration, and myeloid differentiation capacity of BM-MNC $[14,40]$. This age effect was also observed in our study population: a negative relation was found with both GM-CFU and BFU-E numbers; however, this bias was limited since our study cohorts did not differ with respect to age. 
Additionally, in the regression model, analyses were adjusted for age.

Moreover, also medication appears to have its influence on the progenitor cell function and numbers. Statins have shown to stimulate BM-derived EPC survival, differentiation and function, as well as mobilization into the circulation [41]. In addition, non-selective beta-blockers appear to increase HPC growth and proliferation in the $\mathrm{BM}$, although they play a suppressing role on the HPC mobilization [42]. In contrast, $\mathrm{ACE}$ inhibitors stimulate $\mathrm{CD} 34^{+}$cell recruitment into the circulation and towards the place of injury [43].

Additionally, concomitant chronic kidney disease is a wellknown cause of anemia in HF patients and therefore assigned as exclusion criterion in our study [44]. Also patients with diabetes were withdrawn: type I and II diabetes have not only been associated with a reduced number of EPC but also with a reduced VEGF-/SDF- $1 \alpha$-induced migration capacity and proliferation [24]. Moreover, in the setting of cardiovascular disease, the co-presence of diabetes impairs CD34+ mobilization from the BM [25].

\section{Limitations}

Differences in BM-derived progenitor cell function between various underlying etiologies for cardiovascular diseases were evident in our study; yet, our results need to be further confirmed in larger trials.

\section{Conclusions}

The differentiation capacity of BM-derived progenitor cells is reduced in patients with IHD, which may hamper the clinical efficacy of autologous stem cell therapy. This functional impairment worsens with increasing complexity of coronary lesions but is not observed in the setting of non-ischemic cardiomyopathy. These findings suggest that IHD is an important determinant of progenitor cell function in the BM compartment. The observed myelosuppression in IHD is not driven by low-grade systemic inflammation, suggesting that other mechanisms are at play. Future studies should aim to elucidate the exact mechanisms for reduced BM-progenitor cell function in IHD in order to increase the success rate of BM cell therapy in this patient population.

Compliance with Ethical Standards The study complied with the Helsinki Declaration and was approved by the local ethics committee. Informed consent was obtained from all subjects.

Funding The work was supported by a research grant from the Fund for Scientific Research (FWO-Flanders, G060321N). EN is supported by a research grant from the University of Antwerp. EVC is supported by FWO-Flanders as senior clinical investigator.
Competing Interests The authors declare that they have no competing interests.

\section{References}

1. Orlic, D., Kajstura, J., Chimenti, S., Jakoniuk, I., et al. (2001). Bone marrow cells regenerate infarcted myocardium. Nature, 410(6829), 701-705.

2. Lipinski, M. J., Biondi-Zoccai, G. G., Abbate, A., Khianey, R., et al. (2007). Impact of intracoronary cell therapy on left ventricular function in the setting of acute myocardial infarction: a collaborative systematic review and meta-analysis of controlled clinical trials. Journal of the American College of Cardiology, 50(18), 17611767.

3. Delewi, R., Hirsch, A., Tijssen, J. G., Schachinger, V., et al. (2014). Impact of intracoronary bone marrow cell therapy on left ventricular function in the setting of ST-segment elevation myocardial infarction: a collaborative meta-analysis. European Heart Journal, 35(15), 989-998.

4. Tongers, J., Losordo, D. W., \& Landmesser, U. (2011). Stem and progenitor cell-based therapy in ischaemic heart disease: promise, uncertainties, and challenges. European Heart Journal, 32(10), 1197-1206.

5. Fisher, S. A., Doree, C., Mathur, A., \& Martin-Rendon, E. (2015). Meta-analysis of cell therapy trials for patients with heart failurean update. Circulation Research.

6. Afzal, M. R., Samanta, A., Shah, Z. I., Jeevanantham, V., et al. (2015). Adult bone marrow cell therapy for ischemic heart disease: evidence and insights from randomized controlled trials. Circulation Research, 117(6), 558-575.

7. Perin, E. C., Willerson, J. T., Pepine, C. J., Henry, T. D., et al. (2012). Effect of transendocardial delivery of autologous bone marrow mononuclear cells on functional capacity, left ventricular function, and perfusion in chronic heart failure: the FOCUS-CCTRN trial. JAMA, 307(16), 1717-1726.

8. Assmus, B., Fischer-Rasokat, U., Honold, J., Seeger, F. H., et al. (2007). Transcoronary transplantation of functionally competent BMCs is associated with a decrease in natriuretic peptide serum levels and improved survival of patients with chronic postinfarction heart failure: results of the TOPCARE-CHD Registry. Circulation Research, 100(8), 1234-1241.

9. Sant'Anna, R. T., Fracasso, J., Valle, F. H., Castro, I., et al. (2014). Direct intramyocardial transthoracic transplantation of bone marrow mononuclear cells for non-ischemic dilated cardiomyopathy: INTRACELL, a prospective randomized controlled trial. Revista Brasileira de Cirurgia Cardiovascular, 29(3), 437-447.

10. Hamshere, S., Arnous, S., Choudhury, T., Choudry, F., et al. (2015). Randomized trial of combination cytokine and adult autologous bone marrow progenitor cell administration in patients with nonischaemic dilated cardiomyopathy: the REGENERATE-DCM clinical trial. European Heart Journal.

11. Westenbrink, B. D., Voors, A. A., de Boer, R. A., Schuringa, J. J., et al. (2010). Bone marrow dysfunction in chronic heart failure patients. European Journal of Heart Failure, 12(7), 676-684.

12. Kissel, C. K., Lehmann, R., Assmus, B., Aicher, A., et al. (2007). Selective functional exhaustion of hematopoietic progenitor cells in the bone marrow of patients with postinfarction heart failure. Journal of the American College of Cardiology, 49(24), 23412349.

13. Cogle, C. R., Wise, E., Meacham, A. M., Zierold, C., et al. (2014). Detailed analysis of bone marrow from patients with ischemic heart 
disease and left ventricular dysfunction: BM CD34, CD11b, and clonogenic capacity as biomarkers for clinical outcomes. Circulation Research, 115(10), 867-874.

14. Heeschen, C., Lehmann, R., Honold, J., Assmus, B., et al. (2004). Profoundly reduced neovascularization capacity of bone marrow mononuclear cells derived from patients with chronic ischemic heart disease. Circulation, 109(13), 1615-1622.

15. Jakob, P., Doerries, C., Briand, S., Mocharla, P., et al. (2012). Loss of angiomiR-126 and 130a in angiogenic early outgrowth cells from patients with chronic heart failure: role for impaired in vivo neovascularization and cardiac repair capacity. Circulation, 126(25), 2962-2975.

16. Liguori, A., Fiorito, C., Balestrieri, M. L., Crimi, E., et al. (2008). Functional impairment of hematopoietic progenitor cells in patients with coronary heart disease. European Journal of Haematology, 80(3), 258-264.

17. Lin, C. P., Lin, F. Y., Huang, P. H., Chen, Y. L., et al. (2013). Endothelial progenitor cell dysfunction in cardiovascular diseases: role of reactive oxygen species and inflammation. BioMed Research International, 2013, 845037.

18. Pronk, C. J., Veiby, O. P., Bryder, D., \& Jacobsen, S. E. (2011). Tumor necrosis factor restricts hematopoietic stem cell activity in mice: involvement of two distinct receptors. The Journal of Experimental Medicine, 208(8), 1563-1570.

19. Sianos, G., Morel, M. A., Kappetein, A. P., Morice, M. C., et al. (2005). The SYNTAX Score: an angiographic tool grading the complexity of coronary artery disease. EuroIntervention, 1(2), 219-227.

20. D’Agostino, R. B., Sr., Vasan, R. S., Pencina, M. J., Wolf, P. A., et al. (2008). General cardiovascular risk profile for use in primary care: the Framingham Heart Study. Circulation, 117(6), 743-753.

21. Nollet, E., Van Craenenbroeck, E. M., Martinet, W., Rodrigus, I., et al. (2015). Bone matrix vesicle-bound alkaline phosphatase for the assessment of peripheral blood admixture to human bone marrow aspirates. Clinica Chimica Acta, 446, 253-260.

22. Gajkowska, A., Oldak, T., Jastrzewska, M., Machaj, E. K., et al. (2006). Flow cytometric enumeration of CD34+ hematopoietic stem and progenitor cells in leukapheresis product and bone marrow for clinical transplantation: a comparison of three methods. Folia Histochemica et Cytobiologica, 44(1), 53-60.

23. Van Craenenbroeck, E. M., Van Craenenbroeck, A. H., van Ierssel, S., Bruyndonckx, L., et al. (2013). Quantification of circulating $\mathrm{CD} 34+/ \mathrm{KDR}+/ \mathrm{CD} 45 \mathrm{dim}$ endothelial progenitor cells: analytical considerations. International Journal of Cardiology, 167(5), $1688-1695$.

24. Dimmeler, S., \& Leri, A. (2008). Aging and disease as modifiers of efficacy of cell therapy. Circulation Research, 102(11), 1319-1330.

25. Fadini, G. P., \& Avogaro, A. (2013). Diabetes impairs mobilization of stem cells for the treatment of cardiovascular disease: a metaregression analysis. International Journal of Cardiology, 168(2), 892-897.

26. Bozdag-Turan, I., Turan, R. G., Paranskaya, L., Arsoy, N. S., et al. (2012). Correlation between the functional impairment of bone marrow-derived circulating progenitor cells and the extend of coronary artery disease. Journal of Translational Medicine, 10, 143.

27. Coats, A. J. (2001). Heart failure: what causes the symptoms of heart failure? Heart, 86(5), 574-578.

28. Vasa, M., Fichtlscherer, S., Aicher, A., Adler, K., et al. (2001). Number and migratory activity of circulating endothelial progenitor cells inversely correlate with risk factors for coronary artery disease. Circulation Research, 89(1), E1-E7.

29. Valgimigli, M., Rigolin, G. M., Fucili, A., Porta, M. D., et al (2004). CD34+ and endothelial progenitor cells in patients with various degrees of congestive heart failure. Circulation, 110(10), $1209-1212$.

30. Pu, S., Qin, B., He, H., Zhan, J., et al. (2016). Identification of early myeloid progenitors as immunosuppressive cells. Scientific Reports, 6, 23115.

31. Ren, G., Zhang, L., Zhao, X., Xu, G., et al. (2008). Mesenchymal stem cell-mediated immunosuppression occurs via concerted action of chemokines and nitric oxide. Cell Stem Cell, 2(2), 141-150.

32. Chen, X., \& Oppenheim, J. J. (2011). Contrasting effects of TNF and anti-TNF on the activation of effector T cells and regulatory $\mathrm{T}$ cells in autoimmunity. FEBS Letters, 585(23), 3611-3618.

33. Schling, P., Rudolph, C., Heimerl, S., Fruth, S., et al. (2006). Expression of tumor necrosis factor alpha and its receptors during cellular differentiation. Cytokine, 33(5), 239-245.

34. Polz, J., Remke, A., Weber, S., Schmidt, D., et al. (2014). Myeloid suppressor cells require membrane TNFR2 expression for suppressive activity. Immunity, Inflammation and Disease, 2(2), 121-130.

35. Abu-Amer, Y., Erdmann, J., Alexopoulou, L., Kollias, G., et al. (2000). Tumor necrosis factor receptors types 1 and 2 differentially regulate osteoclastogenesis. The Journal of Biological Chemistry, 275(35), 27307-27310

36. Maciejewski, J. P., Kim, S., Sloand, E., Selleri, C., et al. (2000). Sustained long-term hematologic recovery despite a marked quantitative defect in the stem cell compartment of patients with aplastic anemia after immunosuppressive therapy. American Journal of Hematology, 65(2), 123-131.

37. Mangialardi, G., Katare, R., Oikawa, A., Meloni, M., et al. (2013). Diabetes causes bone marrow endothelial barrier dysfunction by activation of the RhoA-Rho-associated kinase signaling pathway. Arteriosclerosis, Thrombosis, and Vascular Biology, 33(3), 555564.

38. Spyridopoulos, I., Erben, Y., Brummendorf, T. H., Haendeler, J., et al. (2008). Telomere gap between granulocytes and lymphocytes is a determinant for hematopoetic progenitor cell impairment in patients with previous myocardial infarction. Arteriosclerosis, Thrombosis, and Vascular Biology, 28(5), 968-974.

39. Nollet, E., Hoymans, V. Y., Van Craenenbroeck, A. H., Vrints, C. J., et al. (2016). Improving stem cell therapy in cardiovascular diseases: the potential role of microRNA. The American Journal of Physiology - Heart and Circulatory Physiology, ajpheart 00239 02016.

40. Schundeln, M. M., Walde, G., Basu, O., Havers, W., et al. (2014). Quantification of nucleated cells, CD34-positive cells and CFUGM colonies in single bone marrow samples and bone marrow harvests derived from healthy children. Pediatric Hematology and Oncology, 31(4), 340-348.

41. Llevadot, J., Murasawa, S., Kureishi, Y., Uchida, S., et al. (2001). HMG-CoA reductase inhibitor mobilizes bone marrow-derived endothelial progenitor cells. The Journal of Clinical Investigation, 108(3), 399-405.

42. Beiermeister, K. A., Keck, B. M., Sifri, Z. C., ElHassan, I. O., et al. (2010). Hematopoietic progenitor cell mobilization is mediated through beta- 2 and beta-3 receptors after injury. The Journal of Trauma, 69(2), 338-343.

43. Cangiano, E., Marchesini, J., Campo, G., Francolini, G., et al. (2011). ACE inhibition modulates endothelial apoptosis and renewal via endothelial progenitor cells in patients with acute coronary syndromes. American Journal of Cardiovascular Drugs, 11(3), 189-198.

44. Babitt, J. L., \& Lin, H. Y. (2012). Mechanisms of anemia in CKD. Journal of the American Society of Nephrology, 23(10), 16311634. 